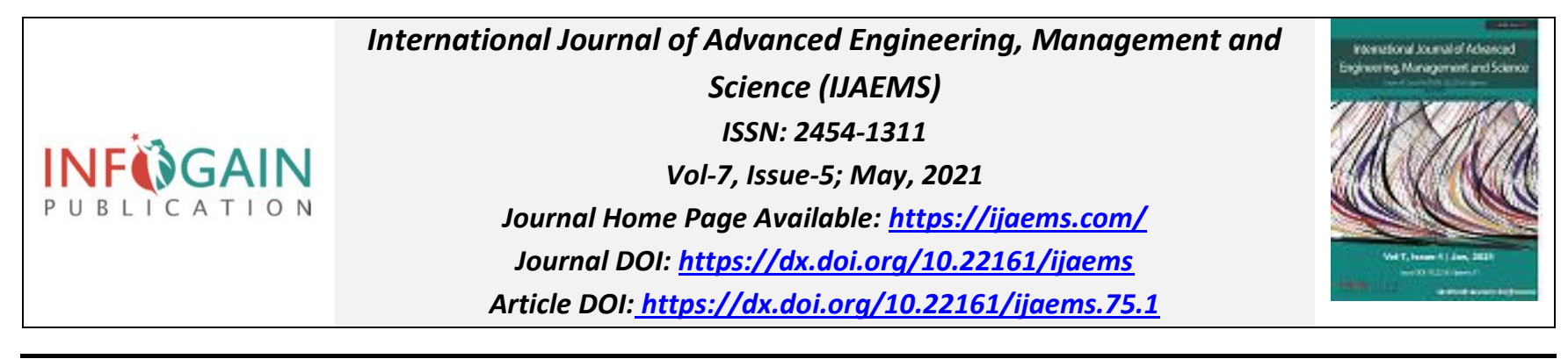

\title{
Recent applications of HPLC in food analysis: A mini review
}

\author{
Mst Nushrat Yiasmin ${ }^{1}$, AL-Ansi Waleed ${ }^{1,2}$ \\ ${ }^{1}$ School of Food Science and Technology, State Key Laboratory of Food Science and Technology, Jiangnan University, 1800 Lihu \\ Avenue, Wuxi 214122, China. \\ ${ }^{2}$ Department of Food Science and Technology, Faculty of Agriculture, Sana'a University, Sana'a, Yemen.
}

Received: 16 Feb 2021; Received in revised form: 10 Mar 2021; Accepted: 19 Apr 2021; Available online: 04 May 2021 (C)2021 The Author(s). Published by Infogain Publication.

\begin{abstract}
Chromatographic separation techniques are multistage separation methods in which the components of a sample are distributed between two phases, of which one is stationary and the other is mobile. High-performance liquid chromatography (HPLC) has emerged as the most powerful and versatile separation and analytical method. High-performance liquid chromatography (HPLC) is a type of liquid chromatography used to separate and quantify compounds that have been dissolved in solution. HPLC is used to determine the amount of a specific compound in a solution. This article is primed with an aim to review different aspects of HPLC such as principle, types, and its application in the food analysis field.
\end{abstract}

Keywords-Chromatography; food analysis; HPLC.

\section{INTRODUCTION}

Analytical chemistry is widely used in determining the qualitative and quantitative composition of typical mixtures. These two methods are the main aspects to understand the sample material. Generally, analytical chemistry divides into two branches i.e. qualitative and quantitative analysis. Therefore, in order to carry on these analyses, an analytical technique termed chromatography was founded. The chromatography term was derived from the Greek words namely chroma (color)) and graphein (to write). Chromatography can be described as an analytical technique utilized for the separation, purification, and identification of constituents from the mixture. It works on the principle of differential interaction of solutes with two different phases, viz., the stationary phase and the mobile phase. Many modifications were made to the techniques of chromatography to overcome the shortcomings like analysis time and the range of compounds that could be detected. The application of pressure was practiced by the use of pumps to reduce the time of a run. Technologies like spectroscopy and electrochemical methods were added to enhance detection. With these developments and modifications, the functional efficiency of chromatographic techniques improved to a great extent and also the range and type of substances that could be analyzed (Ravali et al, 2011).

High-performance liquid pressure chromatography is also known as High-pressure Liquid chromatography (HPLC) is an analysis technique used for separation, identification, and quantification of a typical mixture such as organic and inorganic, biological, ionic, and polymeric materials. HPLC is a type of column chromatography in which solvent flows with high pressure, so that sample can be separated into different constituents divided into different types based on the mode of separation, the principle of separation, elution technique, the scale of separation, and based on the type of analysis (Olander, 1984). Increasingly, food analysis methods are built around high-performance liquid chromatography (HPLC), which has proven to be an optimal technology for detecting and/or quantifying the vast majority of food analytes. These methods employ a stepwise approach that first removes the sample matrix, then isolates the analytes of interest and individually resolves them on a chromatographic column. The efficiency of the separation depends on, among other things, the differential interaction of analytes of interest with both

This article can be downloaded from here: www.ijaems.com

This work is licensed under a Creative Commons Attribution 4.0 License. http://creativecommons.org/licenses/by/4.0/ 
mobile and column stationary phases. Of course, classifying food analytes according to their relative volatility and polarity are factors that must be considered when selecting an appropriate analytical method for their determination. High-pressure liquid chromatography (HPLC) is widely accepted as an invaluable technique for the analysis of many food components. In many instances HPLC methods have replaced laborious analyses and, in general, the chromatographic methods are more specific and precise, coupled with a significant reduction in analysis times.

\section{INSTRUMENTATION AND DATA}

The basic HPLC chromatography consists of a solvent supply, a pump, an injection system, a column, and a detector coupled to a chart recorder. Figure 1 shows a schematic diagram of an HPLC system.

\subsection{SOLVENT RESERVOIR}

In HPLC the mobile phase or solvent is a mixture of polar and non-polar liquid components. Depending on the composition of the sample, the polar and non-polar solvents will be varied (Hopmann, 2011). The solvent reservoir may be any convenient vessel, but provision must be made for degassing the solvent, either by application of vacuum or heat or by ultra-sonification. Failure to degas solvents, particularly protic solvents, may lead to air bubble formation in the detection cell, with consequent disruption of the chromatogram.

\subsection{PUMP}

The pump suctions the mobile phase from the solvent reservoir and forces it to the column and then passes to the detector. $42000 \mathrm{KPa}$ is the operating pressure of the pump. This operating pressure depends on column dimensions, particle size, flow rate, and composition of the mobile phase (Xiang, 2006). Modern HPLC systems have been improved to work at much higher pressures, and therefore be able to use much smaller particle sizes in the columns $(<2$ micrometers).

\subsection{SAMPLE INJECTOR}

An injector for an HPLC framework should give an infusion of the fluid specimen inside the scope of $0.1 \mathrm{~mL}$ to $100 \mathrm{~mL}$ of volume with high reproducibility and under high pressure (up to $4000 \mathrm{psi}$ ) (Simpson, 1976). For liquid chromatography, liquid samples can be directly injected and solid samples need only to be diluted in the appropriate solvent.

Compared with manual injectors, automated sampling systems offer highly reproducible injection volume, low carryover, and increased sample throughput. Modern autosamplers are designed for online sample preparation and derivatization, and in some cases, to safely handle highly corrosive solvents or mobile-phase additives. They may also offer cooling and heating capabilities to preserve unstable species or to induce reactions.

\subsection{COLUMNS}

Columns are typically made of cleaned stainless steel, are somewhere around $50 \mathrm{~mm}$ and $300 \mathrm{~mm}$ long, and have an inward distance across of somewhere around 2 and $5 \mathrm{~mm}$. They are generally loaded with a stationary phase with a molecule size of $3 \mu \mathrm{m}$ to $10 \mu \mathrm{m}$ (Martin and Guiochon, 2005).

\subsection{DETECTOR}

Important detection parameters are dynamic range, calibration linearity, chromatographic selectivity, and qualitative information. A detector is considered selective if it does not respond to co-eluting compounds that could interfere with analyte quantification (Simpson, 1976). The HPLC detector, situated toward the end of the column distinguishes the analytes as they elute from the chromatographic column. Regularly utilized detectors are UV-spectroscopy, fluorescence, mass spectrometric, and electrochemical identifiers. The function of the detector is to examine the solution which is eluting from the column.

\subsection{DATA COLLECTION DEVICES OR INTEGRATOR}

Signals from the detector might be gathered on graph recorders or electronic integrators that fluctuate in manysided quality and in their capacity to process, store and reprocess chromatographic information. The PC coordinates the reaction of the indicator to every part and places it into a chromatograph that is anything but difficult to interpret (Malviya et al, 2010).

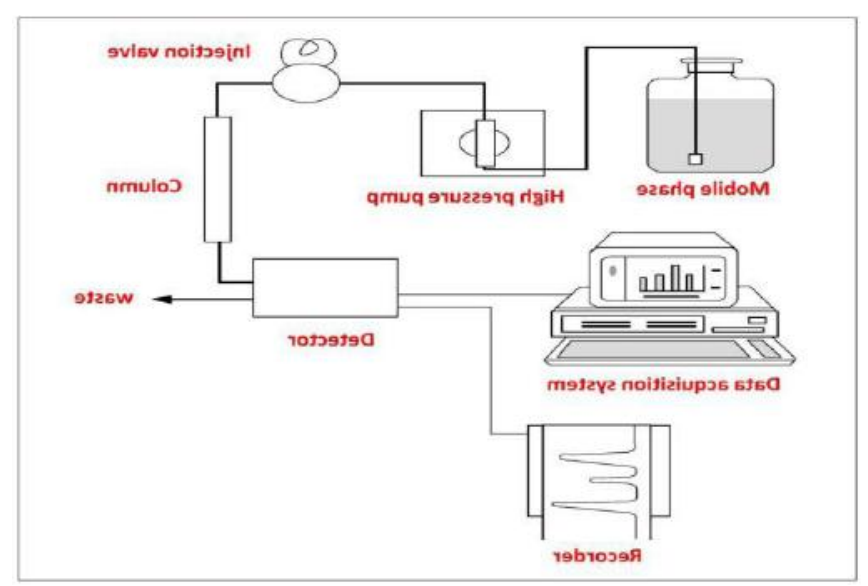

Fig.1: Schematic representation of an HPLC system.

\subsection{PRINCIPLE AND TYPES OF HPLC}

The main principle of separation is adsorption. Factors determining the Resolving power $\left(\mathrm{R}_{\mathrm{s}}\right)$ are 
$\mathrm{R}_{\mathrm{s}}=\frac{\sqrt{ } N}{4}\left(\frac{\alpha-1}{\propto}\right)\left(\frac{\mathrm{k} 2}{1+\text { Kav }}\right)$

Where

- Number of theoretical plates $(\mathrm{N})$ in the column

- Column selectivity $(\alpha)$

- Column retentivity $(\mathrm{k})$

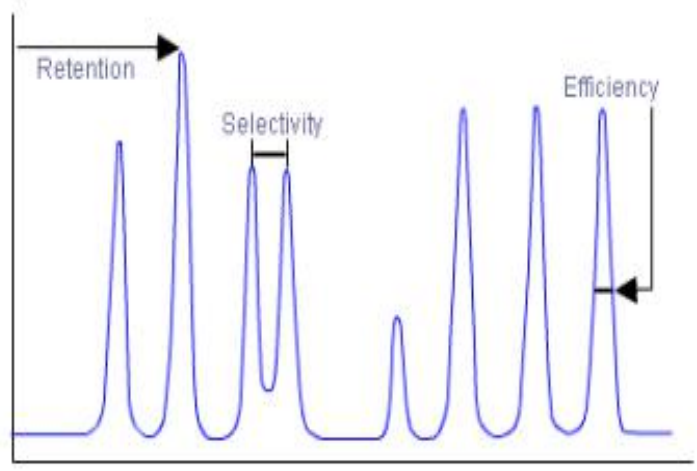

Fig.2: Shows the fundamental parameters

The retention (or capacity) factor $(k)$ is a means of measuring the retention of an analyte on the chromatographic column. A high $k$-value indicates that the sample is highly retained and has spent a significant amount of time interacting with the stationary phase. The retention factor is equal to the ratio of retention time of the analyte on the column to the retention time of a non-retained compound. The non-retained compound has no affinity for the stationary phase and elutes with the solvent front at a time $t_{0}$, which is also known as the 'hold-up time' or 'dead time'

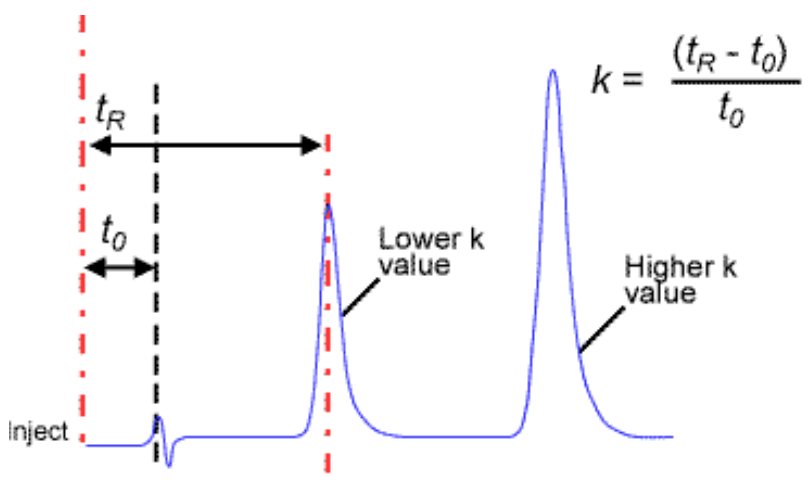

Determination of Retention Factor (k)

Fig.3: Determination of retention factor $(k)$

The selectivity (or separation) factor $(\alpha)$ is the ability of the chromatographic system to 'chemically' distinguish between sample components. It is usually measured as a ratio of the retention (capacity) factors $(\mathrm{k})$ of the two peaks in question and can be visualized as the distance between the apices of the two peaks. High $\alpha$ values indicate good separating power and good separation between the APEX of each peak. However, the alpha value is NOT directly indicative of the resolution. By definition, the selectivity is always greater than one - as when $\alpha$ is equal to one, the two peaks are co-eluting (i.e. their retention factor values are identical). The greater the selectivity value, the further apart the apices of the two peaks become. As the selectivity of separation is dependent upon the chemistry of the analyte, mobile, and stationary phases all of these factors may be altered in order to change or optimize the selectivity of an HPLC separation.

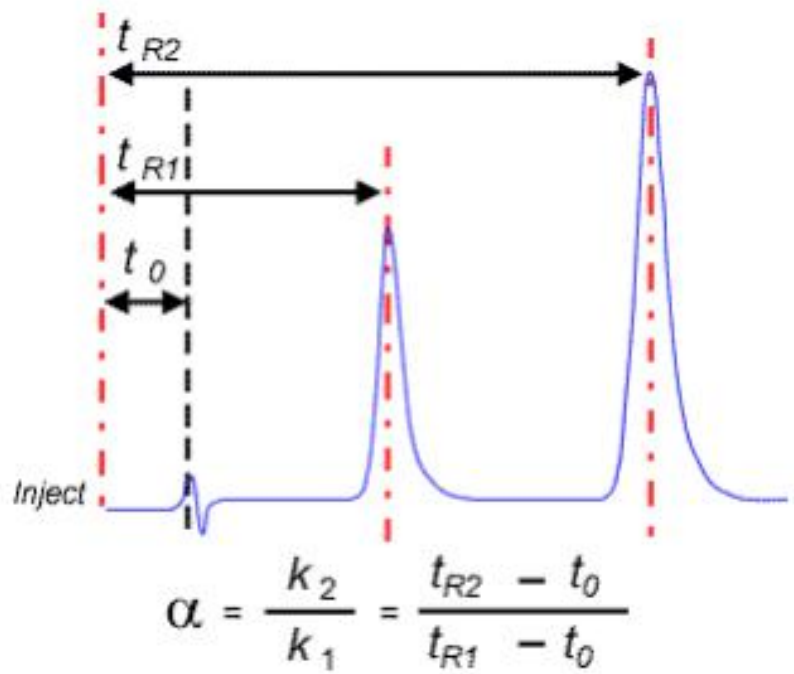

Fig.4: Determination of selectivity $(\alpha)$

The efficiency of a chromatographic peak is a measure of the dispersion of the analyte band as it travels through the HPLC system and column. The plate number $(\mathrm{N})$ is a measure of the peak dispersion on the HPLC column, which reflects the column performance. Efficiency is derived from an analogy of Martyn and Synge who likened column efficiency to fractional distillation, where the column is divided into Theoretical Plates. Each plate is the distance over which the sample components achieve one equilibration between the stationary and mobile phase in the column.

\subsection{TYPES OF HPLC}

Types of HPLC generally depend on the phase system used in the process (Abidi, 1991). Following types are of HPLC generally used in analysis-

\subsubsection{ABSORPTION CHROMATOGRAPHY}

The absorption chromatography solute molecules bond directly to the surface of the stationary phase (Shibasaki, 2012). The liquid-solid interface Molecules are reversibly bound to this surface by dipole-dipole interactions. The 
component which has more affinity towards the mobile phase elutes first and the component which has less affinity towards stationary phase elutes later. No two components have the same affinity towards the mobile phase and stationary phase.

\subsubsection{ION PAIRING CHROMATOGRAPHY}

Ion pairing chromatography is an alternative to ionexchange chromatography. It is a form of chromatography in which ions in solution can be "paired" or neutralized and separated as an ion pair on a reversed-phase column. Mixtures of acids, bases, and neutral substances are often difficult to separate by ion-exchange techniques. In these cases, ion-pairing chromatography is applied. The stationary phases used are the same reversed phases as developed for reversed-phase chromatography. An ionic organic compound, which forms an ion-pair with a sample component of opposite charge, is added to the mobile phase. This ion-pair is, chemically speaking, a salt that behaves chromatographically like a non-ionic organic molecule that can be separated by reversed-phase chromatography.

\subsubsection{CHIRAL CHROMATOGRAPHY}

It involves the separation of stereoisomers. In the case of enantiomers, these have no chemical or physical differences apart from being three-dimensional mirror images. Conventional chromatography or other separation processes are incapable of separating them. To enable chiral separations to take place, either the mobile phase or the stationary phase must themselves be made chiral, giving differing affinities between the analytes (Leon, 2014).

\subsubsection{ION- EXCHANGE CHROMATOGRAPHY}

Ion exchange chromatography is a process that allows the separation of ions and polar molecules based on their charge. It can be used for almost any kind of charged molecule including large proteins, small nucleotides, and amino acids. Retention is based on the attraction between solute ions and charged sites bound to the stationary phase. Ions of the same charge are excluded. The use of resin (the stationary solid phase) is used to covalently attach anions or cations onto it. Solute ions of the opposite charge in the mobile liquid phase are attracted to the resin by electrostatic forces (Malviya, 2010).

\subsubsection{SIZE EXCLUSION CHROMATOGRAPHY (SEC) OR GEL PERMEATION CHROMATOGRAPHY (GPC)}

This type of chromatography lacks an attractive interaction between the stationary phase and solute. Sample molecules small enough to enter the pore structure are retarded, while larger molecules are excluded and therefore rapidly carried through the column. Thus, size exclusion chromatography means the separation of molecules by size.

\subsubsection{REVERSED PHASE CHROMATOGRAPHY}

It is the reverse of the normal phase chromatography in which the stationary phase is non-polar and the mobile phase is polar. An example of the mobile phase is organic solvents (methanol, acetonitrile), buffer (phosphate buffer) (Malviya, 2010).

\subsection{APPLICATION OF HPLC IN FOOD ANALYSIS}

The literature on food analysis can be found in a lot of good reviews. All basic constituents of foodstuffs - proteins, lipids, carbohydrates, and vitamins are amenable to liquid chromatography. Various types of columns and detectors used for those analyses demonstrate the versatility of the technique. Almost any type of food matrix can be extracted in order to identify and quantitate trace amounts of analytes.

\subsubsection{CARBOHYDRATES}

Simple sugars, mono- and disaccharides, can readily be analyzed by gas chromatography after conversion to the more volatile trimethylsilyl derivatives. However, oligosaccharides, for example, raffinose and stachyose, are not so amenable to this technique, and a precision of only $10 \%$ is obtained (Delente \& Ladenburg, 1972). HPLC does not require derivatization prior to analysis with the result that the speed of analysis and precision is improved. The majority of analyses, both for simple sugars and oligosaccharides, use partition chromatography on a silica bonded phase, either with amino functionality. In different studies, HPLC has been used for the analysis of sugars in many food commodities, including fruit juices, beverages wines, and many more. Moreover, HPLC has also been used extensively to study the breakdown of polysaccharides both for the production of syrups and the utilization of carbohydrate wastes.

\subsubsection{LIPIDS}

Both saturated and unsaturated triglycerides have been analyzed. Fats and oils are complex mixtures of triglycerides, sterols, and vitamins. The composition of triglycerides is of great interest in food processing and dietary control. Owing to the low stability of triglycerides containing unsaturated fatty acids, reactions with light and oxygen form hydroperoxides, which strongly influence the taste and quality of fats and oils. Adulteration with foreign fats and the use of triglycerides that have been modified by a hardening process also can be detected through triglyceride analysis.

A number of papers have shown the ability of HPLC to separate cis/trans isomers, for example, the separation of all cis linoleic acid (Kroll \& Mieth, 1977). Also, HPLC has been used to study isomeric mixtures in the analysis of the products of oxidation of unsaturated fatty acid. The above 
studies made it possible to detect the hydroperoxides formed, which would not have been possible at the elevated temperatures required for gas chromatography.

\subsubsection{VITAMINS}

Fat-soluble vitamins, such as vitamins E, D, and A, and water-soluble vitamins, such as vitamins $\mathrm{C}, \mathrm{B} 6, \mathrm{~B} 2, \mathrm{~B} 1$, and B12, have been analyzed. Vitamins are biologically active compounds that act as controlling agents for an organism's normal health and growth. Vitamins generally are labile compounds that should not be exposed to high temperatures, light, or oxygen. HPLC separates and detects these compounds at room temperature and blocks oxygen and light (Nollet, 1992). Through the use of spectral information, UV-visible diode-array detection yields qualitative as well as quantitative data. Another highly sensitive and selective HPLC method for detecting vitamins is electrochemical detection. Figure 5 shows a chromatogram of the analysis of water-soluble vitamins in a vitamin tablet.

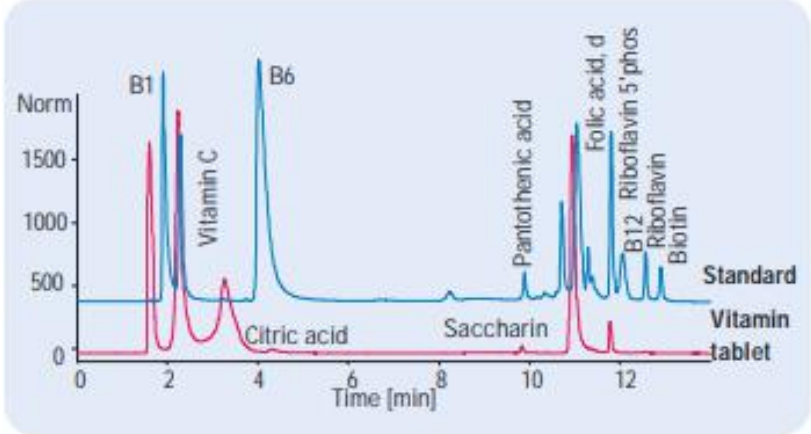

Fig.5: Analysis of water-soluble vitamins in a vitamin tablet

\subsubsection{AMINO ACIDS}

The amino acid composition of proteins can be used to determine the origin of meat products and thus to detect adulteration of foodstuffs. Detection of potentially toxic amino acids is also possible through such analysis. Through the use of chiral stationary phases as column material, D and $\mathrm{L}$ forms of amino acids can be separated and quantified. HPLC in combination with automated online derivatization is now a well-accepted method for detecting amino acids owing to its short analysis time and relatively simple sample preparation.

\section{OTHER APPLICATION OF HPLC IN DIFFERENT INDUSTRIES}

\subsection{PHARMACEUTICAL APPLICATIONS}

- Tablet dissolution study of armaceutical dosages form.
- Shelf-life determinations of pharmaceutical products.

- Identification of active ingredients of dosage forms.

- Pharmaceutical quality control.

\subsection{ENVIRONMENTAL APPLICATIONS}

- Detection of phenolic compounds in Drinking Water.

- Identification of diphenhydramine in sedimented samples.

- Bio-monitoring of pollutants.

\subsection{FORENSICS}

- Quantification of the drug in biological samples.

- Identification of anabolic steroids in serum, urine, sweat, and hair.

- Forensic analysis of textile dyes.

- Determination of cocaine and metabolites in blood.

\section{CONCLUSION}

HPLC has made a significant contribution to the methodology of food analysis and this contribution will doubtless increase in the future. HPLC is a versatile, reproducible chromatographic technique for food analysis. It has wide applications in different fields in terms of quantitative and qualitative estimation of active molecules.

\section{REFERENCES}

[1] Abidi,S.L. High-performance liquid chromatography of phosphatidic acids and related polar lipids. J. Chromatogr. 1991; 587: 193-203

[2] Hearn M.T.W. Ion-pair chromatography on normal and reversed-phase systems. Adv. Chromatogr. 1980; 18: 59100.

[3] Hopmann, E., W. Arlt, and M. Minceva, (2011) Solvent system selection in counter-current chromatography using conductor-like screening model for real solvents. J Chromatogr A, 1218(2): p. 242-50

[4] Leon-Gonzalez, M.E., et al., (2014) Two-dimensional liquid chromatography for direct chiral separations: a review. Biomed Chromatogr. 28(1): p. 59-83

[5] Malviya, R., et al., (2010). High performance liquid chromatography: A short review. Vol. 2. 22-26

[6] Martin, M. and G. Guiochon, (2005). Effects of high pressure in liquid chromatography. J Chromatogr A, 1090(12): p. $16-38$

[7] Nollet, L, M (1992) Food Analysis by HPLC New York

[8] Olander, D.P., (1984). Instrumental Methods of Analysis, Sixth Edition (Willard, Hobart H.; Merritt, Lynne L.; Dean, John A.; Settle, Frank A., Jr.). Journal of Chemical Education. 61(8): p. A222

[9] Ravali R, Phaneendra M, Bhanu JK, Ramya SL, Sushma K. (2011). Recent Trends in Analytical Techniques for the 
Development of Pharmaceutical Drugs. J Bioanal Biomed.; R1: 002.

[10] Shibasaki, H., et al., (2012) Separation and quantitative determination of 6alpha-hydroxycortisol and 6betahydroxycortisol in human urine by high- performance liquid chromatography with ultraviolet absorption detection. Anal Bioanal Chem. 402(9): p. 2945-52

[11] Simpson CF, (1976). Practical High-Performance Liquid Chromatography. London: Heyden and Son;

[12] Xiang, Y., Y. Liu, and M.L. Lee, Ultrahigh pressure liquid chromatography using elevated temperature. J Chromatogr A, 2006. 1104(1-2): p. 198-202 